\title{
Diagnostic and prognostic value of compound motor action potential of lower limbs in acute paraplegic patients
}

\author{
S Rutz ${ }^{1}$, V Dietz ${ }^{1}$ and A Curt*,1 \\ ${ }^{1}$ Swiss Paraplegic Centre, University Hospital Balgrist, Zürich, Switzerland
}

\begin{abstract}
Objectives: To evaluate the diagnostic and prognostic contribution of motor nerve conduction studies (NCS) in addition to neurological examination in patients with acute paraplegia.

Methods: In 79 patients with acute onset of paraplegia due to traumatic or ischaemic damage of the conus medullaris/cauda equina (conus/cauda) or lesion of the mid-thoracic spinal cord (epiconal) neurological (initial and follow-up clinical motor and sensory scores; outcome of ambulatory capacity determined at least 6 months post-trauma) and electrophysiological examinations (motor nerve conduction velocity (MNCV) and compound motor action potential (CMAP) of tibial and peroneal nerves) were performed in parallel.

Results: Severe axonal motor neuropathies were significantly caused by conus/cauda lesions (loss of tibial CMAP in $71 \%$ and of peroneal CMAP in $68 \%$ ) compared to patients with epiconal lesion (no loss of tibial CMAP and abolished peroneal CMAP in 14\%). The CMAPs were deemed acutely pathological 4-14 days post-trauma and were indicative of the severity of conus/cauda lesion while the MNCV remained normal. Follow-up recordings (up to 1 year post trauma) revealed no significant change in the CMAP values. The clinical examination according to the American Spinal Injury Association (ASIA protocol) in contrast to the CMAP values was significantly related to the outcome of ambulatory capacity.

Conclusions: In contrast to patients with an epiconal SCI almost all patients with damage of the conus/cauda present a severe axonal neuropathy of the tibial and peroneal nerves. Pathological CMAPs develop as early as 1-2 weeks after onset of acute paraplegia. They allow, at an early stage, to differentiate between conus/cauda or epiconal lesion and to assess the severity of conus/cauda lesion. Thereafter follow-up examinations remain stable and a developing worsening of peripheral nerve or spinal cord function, eg due to post-traumatic syringomyelia, may be indicated by a secondary deterioration of CMAP values. The clinical examination, according to the ASIA protocol, in acute paraplegia patients, in contrast to the motor nerve conduction studies, is of prognostic value in predicting the outcome of ambulatory capacity.
\end{abstract}

Spinal Cord (2000) 38, 203-210

Keywords: motor nerve conduction study; paraplegia; diagnosis; prognosis

\section{Introduction}

Depending on the neurological level of lesion, acute paraplegic patients who have suffered a spinal cord injury (SCI) or lesion of the conus medullaris/cauda equina, either traumatic or non-traumatic, may develop a spastic or flaccid motor disorder of the legs. Clinical examination of chronic SCI patients can differentiate between mid-thoracic lesion of the spinal cord with a spastic paraparesis (above the conus medullaris, termed here an epiconal lesion) and/or

*Correspondence: A Curt, Swiss Paraplegic Centre, University Hospital Balgrist, Forchstrasse 340, CH 8008 Zürich, Switzerland damage of the conus medullaris and cauda equina resulting in a flaccid and atrophic muscle paresis (termed here conus/cauda lesion). However, the clinical examination of acute paraplegic SCI patients with level of lesion at the thoraco-lumbar junction is of limited value because of spinal shock with abolished tendon reflexes and flaccid leg muscle tone. ${ }^{1}$ In contrast to the lower limb reflexes, the bulbocavernosus reflex (BCR), which is mediated by the sacral roots and cord, is maintained in acute SCI patients with preserved conus/cauda function. The BCR can be evoked within $24 \mathrm{~h}$ post-trauma and precedes re-establishment of the tendon reflexes. ${ }^{2}$ 
Therefore, if the BCR is present in acute SCI patients severe conus/cauda lesion can be discounted. However, the BCR per se cannot evaluate the degree of impaired conus/cauda function. ${ }^{3}$

Electromyographic (EMG) recordings are the most sensitive diagnostic tool available in the assessment of lesions of the peripheral motor nerves, ie from the anterior horn cells located in the conus medullaris and the peripheral motor nerve fibres of the cauda equina. The main disadvantage of this technique is, that EMG is less sensitive in quantifying the degree of damage to peripheral motor nerve fibres in the acute lesion. Furthermore, a period of up to 20 days post trauma is needed for the development of the characteristic EMG signs of such a lesion. ${ }^{4}$

In a previous study, compound motor action potentials (CMAPs) of median and ulnar nerves in tetraplegic SCI patients were of diagnostic and prognostic value in assessing the extent of intramedullary damage to motor nerve fibres and predicting the outcome of hand function. ${ }^{5}$

By recording CMAPs of tibial and peroneal motor nerves in patients with paraplegia the extent and severity of a lesion of intramedullary anterior horn cells of the conus medullaris and motor nerve fibres of the cauda equina due to traumatic/nontraumatic damage can be quantitatively assessed. ${ }^{6}$ However, the time course and the characteristic of pathological motor nerve conduction studies and its diagnostic and prognostic significance in acute paraplegic patients has not yet been evaluated.

The aim of this study in acute paraplegic patients was to assess; (1) the diagnostic significance of motor nerve conduction studies (NCS) of lower limb nerves in epiconal and conus/cauda lesion; (2) the extent and time course of development of pathological CMAPs after lesion from acute to chronic stages of paraplegia; and (3) the prognostic value of CMAPs with respect to the outcome of ambulatory capacity.

\section{Methods}

This study describes data taken from paraplegic patients who were resident in our Rehabilitation Centre between 1993 and 1996. Each patient had suffered either a traumatic or non-traumatic (eg ischaemic) acute paraplegia with a neurological level of lesion between T4 and L4. Approval of the local Ethics Committee was gained and each patient gave their informed consent to participate in the study. Patients with history or signs of pre-existing neuropathy (diabetes mellitus, alcoholism etc.), local nerve entrapments (peroneal nerve compression) or peripheral nerve lesions of the lower limbs were excluded. To assess the diagnostic and prognostic value of motor nerve conduction studies and the time course of respective changes in acute paraplegia two main calculations were performed.
Diagnostic and prognostic value of motor nerve conduction studies

For correlation between both the extent of the neurological deficits (as assessed by the ASIA scores) and the degree of functional outcome (ambulatory capacity) and the acute and follow-up electrophysiological recordings values of patients with epiconal or conus/cauda lesion were calculated. In this prospectively designed group 25 acute paraplegic patients (age $37 \pm 15$ years (mean $\pm \mathrm{SD}$ ); eight female, 17 male; 23 traumatic, two ischaemic) were consecutively included and underwent clinical (neurological deficits) and electrophysiological examinations at three times following the injury. The first examination was performed after admission to the rehabilitation centre following the onset of paraplegia (median 24 days, range $2-50$ days after incidence). This constituted the acute examination. The second examination was at a sub-acute stage (median 117 days, range 54-136 days after onset of paraplegia), which was performed usually at the end of the rehabilitation programme. The final examination was performed at a very chronic stage (median 384 days, range 191-962 days after onset of paraplegia). To assess the diagnostic value of the electrophysiological recordings the clinical examination was aimed to categorise the patients into one of two most relevant clinical SCI syndromes, those with clinically evident conus/cauda lesion (lesion of lower motoneurones, 14 patients; five female, nine male; level of lesion T9-L4) or an epiconal lesion (lesion of upper motoneurones, 11 patients; three female, eight male; level of lesion T4-L1). For calculation of the prognostic significance the recovery of sensory and motor deficits were scored in parallel to the electrophysiological recordings while the outcome of ambulatory capacity was assessed at the end of the rehabilitation programme (the chronic stage of SCI).

Time course of pathologic electrophysiological changes To assess the very early time course of the development of pathologic electrophysiological recordings in acute paraplegia data of 54 patients (mean age $38 \pm 17$ years; 12 female, 42 male; 46 traumatic, eight ischaemic) were calculated, who in the initial and/or at least in the follow-up recordings showed pathological values of the tibial and/or peroneal nerves. The recordings in these patients were deliberately performed at very acute stages and the initial recordings could be taken between $2-57$ days after onset of the acute paraplegia.

\section{Clinical examination}

The clinical differentiation between an epiconal SCI lesion versus a damage of the conus/cauda was achieved by combined neurological and urodynamical examinations. These methods represent the usual clinical approach to differentiate between these two disorders. To prevent misdiagnosis in early stage of acute paraplegia, ie due to spinal shock or recovery 
from transient neurological deficits, the final clinical classification was determined at the chronic stage of paraplegia (at least 6 months after onset).

An epiconal SCI lesion was diagnosed if three conditions were satisfied; if lower-limb tendon reflexes and BCR could be elicited, if normal or increased muscle tone of the legs was present, and in addition, if urodynamic examination revealed a neurogenic bladder dysfunction of upper motoneurone lesion (UMN) (with hyperreflexia of the detrusor muscle, detrusorsphincter dyssynergia). If any of the above conditions was absent an at least incomplete conus/cauda lesion was diagnosed.

Neurological examinations of all patients were performed according to the ASIA standards by specially trained physicians working full-time in the rehabilitation centre under supervision of a senior colleague. ${ }^{7}$ They were performed in parallel with electrophysiological examinations. From the ASIA protocol the following measures were assessed and analyzed separately: (1) ASIA motor score; (2) ASIA pin-prick score; and (3) ASIA light touch score.

The outcome of ambulatory capacity of patients was assessed and attributed to one of four categories. (1) No ambulatory capacity where the patient is unable to walk or stand. (2) Therapeutic ambulatory capacity where standing and walking is possible but only with the support of either two physiotherapists, with two braces in parallel bars or with two sticks and the help of an accompanying person. These patients could walk only as a therapeutical procedure. (3) Functional ambulatory capacity where daily walking was possible over short distances without the aid of physiotherapists or braces. This category describes that walking which would be encountered in daily life or at work. (4) Full ambulatory capacity where there was little or no disturbance in walking. This categorisation corresponds to that used in an earlier study. ${ }^{8}$

\section{Motor nerve conduction studies}

A Dantec EMG 12 electromyograph was used to perform the motor nerve conduction studies from temperature-controlled skin. Supramaximal, rectangular wave stimuli of $(0.2 \mathrm{~ms}$ duration) were applied to the tibial and peroneal nerves distally at the ankle and proximally at the knee. The motor nerve potentials were recorded via surface electrodes (Dantec Silver Chlor Disc Electrode) placed over the m. abductor hallucis brevis and the $\mathrm{m}$. extensor digitorum brevis. The signals were bandpass-filtered between $10 \mathrm{~Hz}$ and $10 \mathrm{kHz}$. The latency and amplitude of the compound motor action potentials (CMAPs, baseline to peak) and the motor nerve conduction velocities (MNCV) of both nerves were calculated. Normal values were set at levels described elsewhere. ${ }^{9}$ The tibial CMAP was classified as pathological below $5.0 \mathrm{mV}$ and the MNCV below $40.6 \mathrm{~m} / \mathrm{s}$; the peroneal CMAP below $4.0 \mathrm{mV}$ and MNCV below $41.7 \mathrm{~m} / \mathrm{s}$.

\section{Statistics}

Statistical analysis was performed using the SAS software package. The relationship of electrophysiological examinations, neurological deficits and outcome of ambulatory capacity was calculated by Spearman correlation analysis (level of significance $P<0.05$ ). The relationship between epiconal and conus/cauda lesion versus ambulatory capacity and motor scores was analyzed with Kruskal-Wallis-Chi square approximation tests. Student's $t$-tests were performed to compare follow up measurements.

\section{Results}

\section{Neurological deficit}

Severe neurological deficits and functional impairment was evident in all the paraplegic patients with either epiconal or conus/cauda lesion upon the initial examination. These deficits persisted in subsequent follow-up examinations. The extent of neurological deficits is reflected in the reduced ASIA lower-limb motor and sensory scores for the epiconal and conus/ cauda lesion patients groups (Table 1). Both the motor and sensory functions were similarly affected (KruskalWallis-Chi square approximation test; $P>0.05)$ in both patients groups.

Table 1 Mean $( \pm$ SD) ASIA score parameters investigated in patients with epiconal or conus/cauda lesion at three stages in the course of paraplegia

\begin{tabular}{llcccc}
\hline Type of lesion & \multicolumn{1}{c}{$\begin{array}{c}\text { ASIA scores } \\
\text { (normal) }\end{array}$} & Acute & $\begin{array}{c}\text { Examination } \\
\text { Sub-acute }\end{array}$ & Chronic & \% \\
\hline Epiconal & motor (100) & $63 \pm 17$ & $65 \pm 19$ & $71 \pm 21$ & 12.7 \\
$(n=11)$ & pin prick (112) & $74 \pm 24$ & $76 \pm 25$ & $80 \pm 24$ & 8.1 \\
& light touch (112) & $78 \pm 21$ & $78 \pm 21$ & $83 \pm 22$ & 6.4 \\
Conus/cauda & motor (100) & $56 \pm 15$ & $62 \pm 16$ & $64 \pm 17$ & $8.3^{*}$ \\
$(n=14)$ & pin prick (112) & $82 \pm 13$ & $84 \pm 13$ & $86 \pm 14$ & 4.9 \\
& light touch (112) & $86 \pm 12$ & $87 \pm 13$ & $88 \pm 14$ & 2.3 \\
\hline
\end{tabular}

*Increase significant; $P<0.01$ 
In the follow-up examinations both patients groups showed an increase in the motor and sensory scores. However, this increase was significant (Student's $t$-test; $P<0.01)$ only for the ASIA motor scores of the conus/ cauda patient group. There was a trend toward recovery of sensory deficits (pin prick and light touch sensation) in both patients groups but the increment was not significant.

\section{Outcome of ambulatory capacity}

The degree of recovery of ambulatory capacity assessed at the end of the rehabilitation programme was similar regardless of the type of lesion (Kruskal-Wallis-Chi square approximation test; $P>0.05)$. As shown in Table 2 according to the severe SCI no patient achieved full ambulatory capacity, $9 \%$ and $7 \%$ of the epiconal and conus/cauda patients respectively regained a therapeutic locomotor capacity. Functional capacity was attained in $45.5 \%$ of epiconal- and $50 \%$ of conus/cauda-lesioned patients. The remainder from each group remained incapable of locomotion. The degree of neurological impairment in both patients groups assessed by the ASIA scores was significantly related to the outcome of ambulatory capacity achieved at the end of the rehabilitation programme (Spearman correlation analysis; $P<0.01$ in epiconal and $P<0.05$ in conus/cauda lesion).

\section{Diagnostic and prognostic value of motor nerve conduction studies}

In the initial examination the mean CMAP values of both peroneal and tibial nerves were pathologically reduced in patients with conus/cauda lesion (Table 3 ).

Table 2 Outcome of ambulatory capacity achieved of the patients (in \%) with epiconal and conus/cauda lesion

\begin{tabular}{lcccc}
\hline $\begin{array}{l}\text { Type of lesion } \\
\text { \% of patients }\end{array}$ & \multicolumn{4}{c}{ Outcome of ambulatory capacity } \\
None & Therapeutic Functional & Full \\
\hline $\begin{array}{l}\text { Epiconal } \\
(n=11)\end{array}$ & 45.5 & 9 & 45.5 & 0 \\
$\begin{array}{l}\text { Conus/cauda } \\
(n=14)\end{array}$ & 43 & 7 & 50 & 0 \\
\hline
\end{tabular}

The CMAP values were significantly (Kruskal-WallisChi square approximation test; $P<0.05)$ less deteriorated in the patients with an epiconal lesion although the mean values of the peroneal CMAPs were pathologically reduced and the mean tibial CMAPs were of low amplitude.

In the acute stage of conus/cauda lesion normal CMAP values were evident in only two of 28 peroneal nerve recordings $(7 \%)$ and in four of 28 (14\%) tibial nerve recordings (see Figure 1 for the initial examination). A complete loss was present in $71 \%$ and in $68 \%$ of peroneal and tibial CMAP recordings respectively. In contrast to the patients with conus/ cauda lesion in patients with epiconal lesion there was no loss of tibial nerve CMAP in any epiconal lesion patient and in only $14 \%$ of patients peroneal nerve CMAP were abolished. However some CMAP reduction was even present in epiconal SCI lesion although the patients did not show any other clinical or urodynamical sign of conus/cauda involvement. During the follow-up recordings (sub-acute and chronic stages) there was a trend toward further CMAP reduction especially in the conus/cauda patients although these changes were not significant $(P>0.05)$ in either group. In contrast to the CMAP reduction the mean values for the motor nerve conduction velocity (MNCV) were within normal limits in both patients groups (Table 3).

The motor nerve conduction studies in neither lesion group, however, did not show a significant correlation (Spearman correlation analysis, $P>0.1$ ) between the initial electrophysiological recordings and the recovery of neurological deficit (ASIA motor and sensory scores) and the outcome of ambulatory capacity assessed at the end of the rehabilitation programme.

Time course of pathological NR

In order to study the time-course of the deterioration of the CMAP we selected those patients $(n=54)$ who at least in the follow-up examinations showed pathological CMAP values in one of the four leg nerves (right and left peroneal and tibial nerves). The initial examination was recorded within a range of 2-57 days after onset of acute paraplegia while the follow-up recordings were performed in a range of 83-191 days

Table 3 Results of the initial and follow-up electrophysiological recordings (mean \pm SD) from patients with epiconal or conus/ cauda lesion

\begin{tabular}{|c|c|c|c|c|c|c|c|}
\hline Type of lesion & Parameters & Acute & $\begin{array}{l}\text { Peroneal nerve } \\
\text { Sub-acute }\end{array}$ & Chronic & Acute & $\begin{array}{l}\text { Tibial nerve } \\
\text { Sub-acute }\end{array}$ & Chronic \\
\hline $\begin{array}{l}\text { Epiconal } \\
(n=22) \\
\text { Conus/cauda } \\
(n=28)\end{array}$ & $\begin{array}{l}\text { CMAP }(\mathrm{mV}) \\
\text { MNCV }(\mathrm{m} / \mathrm{s}) \\
\text { CMAP }(\mathrm{mV}) \\
\text { MNCV }(\mathrm{m} / \mathrm{s})\end{array}$ & $\begin{array}{r}1.9 \pm 1.9 \\
43.9 \pm 4.3 \\
0.5 \pm 1.3 \\
45.2 \pm 5.3\end{array}$ & $\begin{array}{r}1.8 \pm 2.0 \\
43.9 \pm 3.4 \\
0.1 \pm 0.3 \\
4.6 .3 \pm 6.0\end{array}$ & $\begin{array}{r}1.6 \pm 1.7 \\
42.9 \pm 5.3 \\
0.2 \pm 0.4 \\
42.9 \pm 6.9\end{array}$ & $\begin{array}{r}5.8 \pm 4.0 \\
45.6 \pm 4.7 \\
1.3 \pm 3.1 \\
42.0 \pm 6.1\end{array}$ & $\begin{array}{r}5.1 \pm 3.4 \\
43.4 \pm 2.6 \\
0.6 \pm 1.6 \\
43.9 \pm 4.4\end{array}$ & $\begin{array}{r}4.6 \pm 3.4 \\
43.6 \pm 3.7 \\
0.7 \pm 2.0 \\
44.7 \pm 8.6\end{array}$ \\
\hline
\end{tabular}

$n=$ number of nerves recorded 


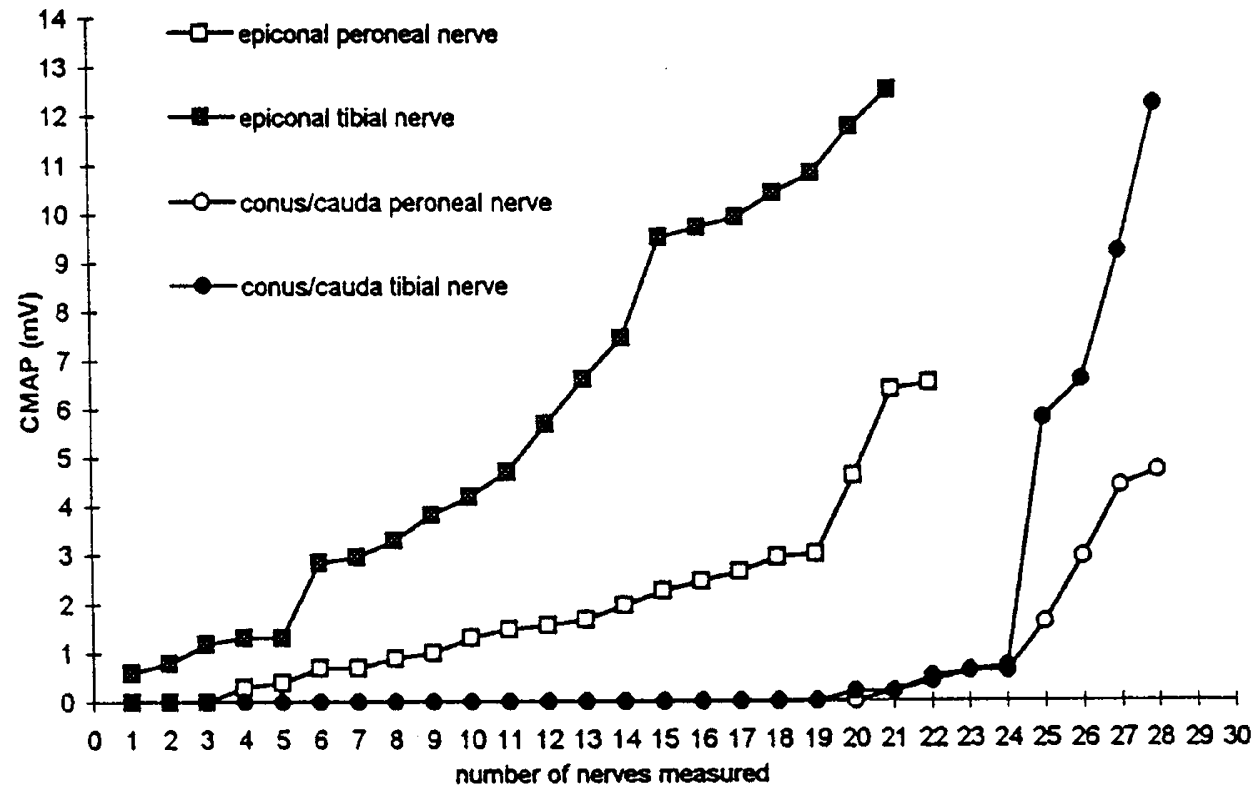

Figure 1 Ranked CMAP values of patients with either epiconal ( $n=11$ patients) or conus/cauda lesion ( $n=14$ patients; recordings were taken from both the right and left nerves)

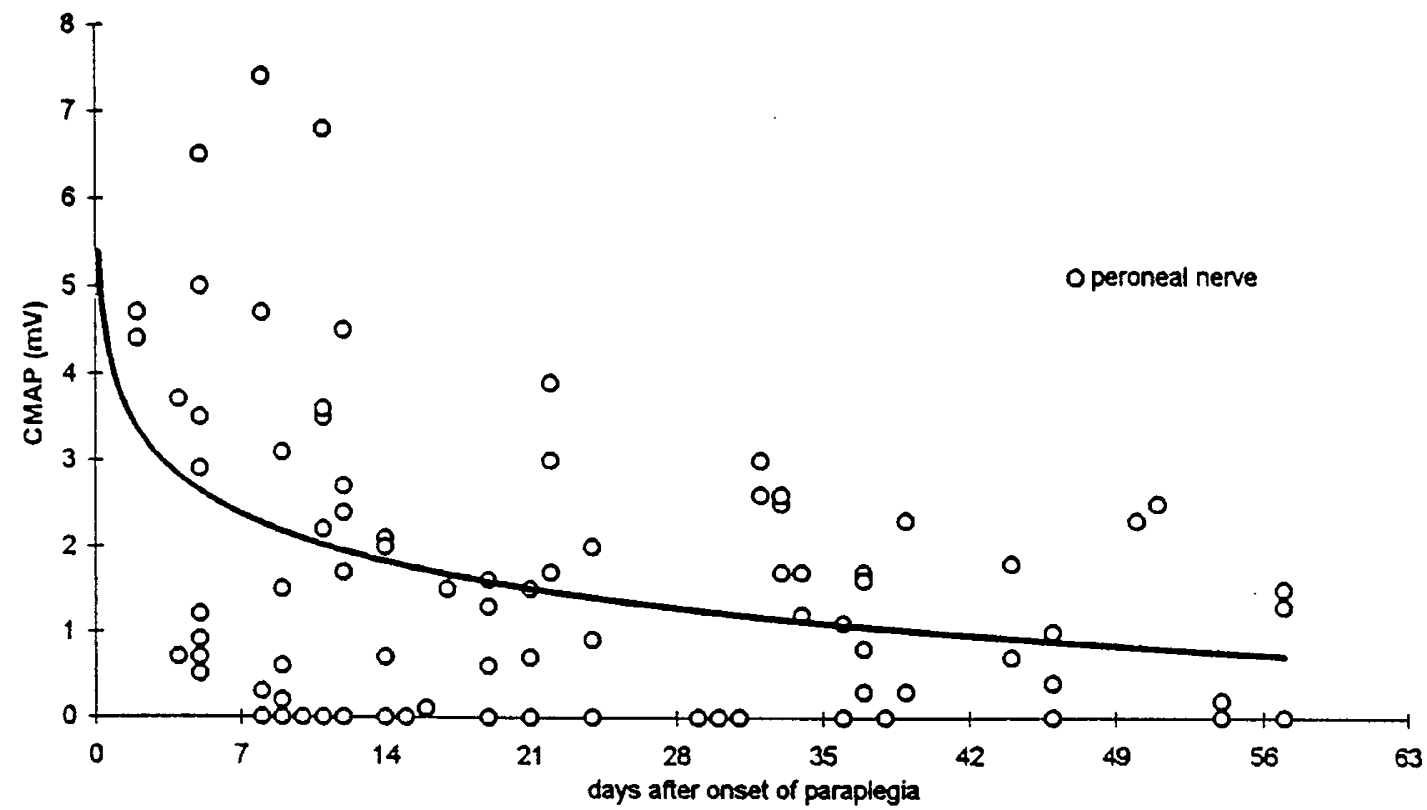

Figure 2 Time course of early CMAP deterioration in acute paraplegia of the peroneal nerve, data were fit by a decaying exponential function (trendline: logarithm order 2 of peroneal nerve values)

after onset of paraplegia. Twenty-six patients had an epiconal lesion and 28 suffered a conus/cauda lesion. The total of 216 nerve recordings showed that the peroneal nerves $(107 / 108)$ were significantly (Student's $t$-test; $P<0.05)$ more affected than the tibial nerves $(82 /$
108). Only a small number of the patients who in follow-up examination revealed pathologically reduced CMAP values showed initially normal CMAP values $(6.5 \%$ of the peroneal and $4 \%$ of the tibial nerves). The deterioration of CMAP values appeared early after 


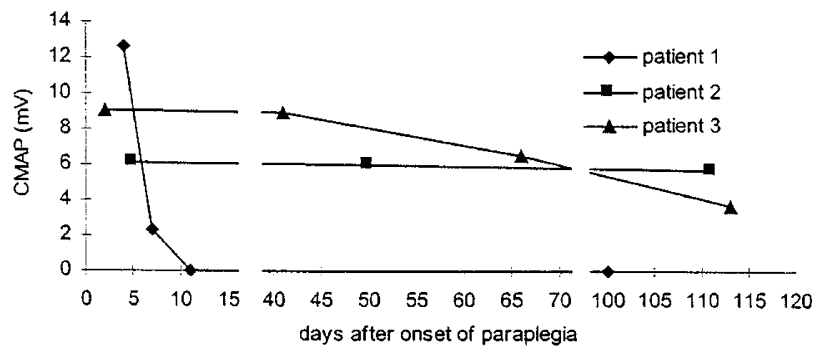

Figure 3 Typical results of CMAP follow-up measurements in acute paraplegic patients with conus/cauda (patient 1) and epiconal lesion (patient 2). Patient 3 however, with an epiconal lesion, showed a delayed worsening of spinal cord function, which was indicating the development of a posttraumatic syringomyelia

lesion and normal values could only be observed in those patients whose initial examination was performed within 14 days after acute onset of paraplegia. Figure 2 demonstrates the time course of early peroneal CMAP deterioration after trauma. Severely reduced CMAPs were already present 4 days post lesion and even totally abolished CMAPs 8 days respectively. In mean pathologically reduced CMAP values of the peroneal developed between 4 and 11 days and of tibial nerves between 4 and 14 days after lesion.

Figure 3 illustrates three characteristic follow-up examinations in acute paraplegic patients. Patient 1 shows the very early deterioration of a tibial CMAP in a patient with an acute conus/cauda lesion. At the initial examination (day 4 post trauma) the tibial CMAP is of normal value but shows a rapid reduction of the CMAP from day 7 to day 11 post trauma with finally complete loss of the CMAP. In contrast, patient 2 with an epiconal acute SCI lesion shows throughout the follow-up examinations almost constant values of tibial CMAP with only a slight reduction. Patient 3, who also suffered an epiconal acute SCI lesion as patient 2, shows in the follow-up examinations a slowly worsening of the CMAP values. This CMAP reduction developed over 3 months post trauma and was not related to the acute spinal cord lesion, but the further neuroradiological examination revealed the development of a post-traumatic syringomyelia.

\section{Discussion}

In patients suffering acute SCI the clinical examination is of essential diagnostic and prognostic value. ${ }^{10,11}$ Although the initial neurological examination determines the level and extent of neurological deficits it can be difficult in injuries at the thoraco-lumbar spine to distinguish between a lesion of the epiconal spinal cord or damage of conus/cauda in acute paraplegic patients. Due to spinal shock in acute traumatic/non-traumatic paraplegia lower limb reflexes are abolished and muscle tone is reduced in both epiconal and conus/cauda lesion. ${ }^{2}$ However, the differentiation between the two conditions is of relevance for the prognosis and treatment of the patient's deficits. In conus/cauda lesion the patient will develop a flaccid, atrophic muscle paresis with neurogenic bladder dysfunction due to impaired lower spinal cord motoneurones (areflexive detrusor muscle function). ${ }^{12}$ In contrast, an epiconal lesion is associated with spastic paraparesis and bladder dysfunction due to impairment of upper motoneurones.

The purpose of this study was to determine the degree to which motor NCS of tibial and peroneal nerves can contribute to the clinical diagnosis and prognosis of acute paraplegic patients. The main findings from our study were: (1) Patients with epiconal and conus/cauda lesion present a similar extent of neurological deficits in the initial examination and outcome of ambulatory capacity. (2) CMAP values are of diagnostic value for differentiation between conus/cauda and epiconal lesion. (3) The peroneal nerves are more affected than the tibial nerves and pathological CMAP values develop within 4-14 days after lesion, thereafter they remain stable. (4) The clinical examination, in contrast to the CMAP values, is of prognostic value for the prediction of ambulatory outcome.

The neurological examination of patients with conus/cauda or epiconal lesion show that the extent of motor and sensory deficits can be very similar in both types of lesion. Although the identification of the neurological level of lesion indicates a conus/cauda or epiconal lesion it can be difficult, ie in lesions of the thoraco-lumbal junction, to distinguish between these types of disorders. Therefore, there is a need for an additional diagnostic tool to the clinical examination which is able to determine the extent of conus/cauda damage early after injury.

In almost all patients with acute conus/cauda lesion the motor NCS of both lower limb nerves were affected and the recordings revealed typical findings of a severe axonal motor neuropathy (reduction or even loss of CMAP, while the MNCV remained normal). The motor NCS in epiconal lesion were significantly less affected. However in some patients with clinically diagnosed epiconal lesion (ie present lower limb reflexes, preserved muscle tone and hyperreflexive neurogenic bladder dysfunction) the CMAP values of the peroneal nerves were pathologically reduced. This shows that even in patients with no clinical signs of conus/cauda lesion MCAP values can be deteriorated revealing a subclinical involvement of lower motoneuron function. The results obtained here in patients with epiconal lesion are in accordance with earlier reports in SCI patients. ${ }^{13-15}$ In electromyography and NCS signs of denervation and neuropathy in muscles below the neurological level of spinal cord injury were seen. ${ }^{16-18}$ This was attributed to a transsynaptic neuronal degeneration of lower motoneurones, due to a loss of trophic influence from upper motoneurones. ${ }^{19-21}$ However, in the latter studies no early electrophysiological recordings were performed, therefore a direct comparison to our study cannot be made. ${ }^{22-24}$ 
The results presented here would favour the assumption of Berman et $a l^{25}$ who proposed that in SCI a neuronal impairment exists not only at the level of injury but over a more extensive area within the spinal cord with the injury level as its rostral border. $^{26-28}$ The time course of pathological CMAP values support this assumption. Pathological CMAP values developed within 4-14 days post-trauma and did not change significantly in the follow-up recordings. This corresponds to results which were obtained in a recent study on tetraplegic patients due to cervical SCI. ${ }^{5}$ About half of the median and ulnar nerves showed a pathological CMAP within the first 2 weeks post-trauma and the time course was found to be similar. We suggest that pathological CMAP values in acute epiconal lesion are also most probably caused by the trauma itself and not due to effects of chronic spinal cord damage. The initial pathological CMAP values can be distinguished from secondary deterioration due to developing peripheral nerve or spinal cord dysfunction. Such a secondary neuropathy was evident in one patient whose CMAP values worsened 3 months post-trauma due to a developing syringomyelia. Although needle EMG recordings are most sensitive to assess an acute peripheral motor nerve lesion, the present study is able to show that CMAP recordings can be used earlier to indicate the extent of conus/cauda damage than EMG, which represents typical characteristics with a time delay of about 20 days post-trauma. ${ }^{29-31}$ The use of $\mathrm{F}$-wave recordings in acute SCI to diagnose conus/cauda damage is restricted due to the influence of spinal shock. ${ }^{32}$

The initial/acute ASIA scores of paraplegic patients with conus/cauda or epiconal lesion were significantly related to the recovery of ambulatory capacity at the end of the rehabilitation programme. This corresponds to findings of earlier SCI studies. ${ }^{33-35}$ In contrast, the initial CMAP values were not of predictive value for the extent of neurological recovery or outcome of ambulatory capacity. This less prognostic value can be explained by the fact that in paraplegic patients the thigh muscles are more relevant for the outcome of ambulatory capacity than the lower leg muscles supplied by the nerves tested in this study. Even patients with complete paraplegia of the lower limbs but preserved thigh muscles can achieve functional capacity with the aid of braces. Therefore these patients showed a similar degree of outcome of ambulatory capacity as did patients with incomplete lesion of lower limb muscles.

We conclude that the clinical examination in acute paraplegia patients is most sensitive to predict the recovery of ambulatory capacity, while motor nerve conduction studies constitute a valuable diagnostic tool to differentiate between epiconal and conus/cauda lesion. CMAP recordings of lower limb motor nerves allow at an early stage to assess and quantify the extent of conus/cauda lesion. Furthermore, follow-up recordings can be used to indicate a secondary impairment of peripheral nerve and spinal cord function.

\section{Acknowledgements}

We thank Dr I Gibson for editorial service, E Harsch for technical assistance and Th Erni (biostatistician) for statistical support. This work was supported by the Swiss National Science Foundation (Grant No. 3200-052562.97) and the International Research Institute for Paraplegia (P38/97).

\section{References}

1 Guttmann L. Spinal shock and reflex behaviour in man. Paraplegia 1970; 8: $100-110$.

2 Rossier AB, Fam BA, Dibenedetto M, Sarkaratti M. Urodynamics in spinal shock. Urol Res 1979; 122: $783-787$.

3 Koyanagi T, Arikado K, Takamatsu T, Tsuji I. Experience with electroymography on the external urethral sphincter in spinal cord injury patients. $J$ Urol 1982; 127: $272-276$.

4 Parry GJ. Electrodiagnostic studies in the evaluation of peripheral nerve and brachial plexus injuries. Neurol Trauma 1992; 4: 921 - 934.

5 Curt A, Dietz V. Nerve conduction study in cervical spinal cord injury: significance for hand function. Neuro Rehabilitation 1996; 7: $165-173$.

6 Boltshauser E, Isler W, Bucher HU, Friderich H. Permanent flaccid paraplegia in children with thoracic spinal cord injury. Paraplegia 1981; 19: 227 -234.

7 Maynard Jr MF et al. International standards for neurological function classification of spinal cord injury. Spinal Cord 1997; 35: $266-274$.

8 Curt A, Dietz V. Ambulatory capacity in spinal cord injury: significance of somatosensory evoked potentials and ASIA protocol in predicting outcome. Arch Phys Med Rehabil 1997; 78: $39-43$.

9 Robinson LR et al. Influences of height and gender on normal nerve conduction studies. Arch Phys Med Rehabil 1993; 74: $1134-1138$.

10 Waters RL, Rodney A, Yakura JS, Vigil D. Prediction of ambulatory performance based on motor scores derived from standard of the American Spinal Injury Association. Arch Phys Med Rehabil 1994; 75: 750-767.

11 Iseli E, Cavigelli A, Dietz V, Curt A. Prognosis and recovery in ischaemic and traumatic spinal cord injury: clinical and electrophysiological evaluation. J Neurol Neurosurg Psychiatry 1999; 67: $567-571$.

12 Curt A, Rodic B, Schurch B, Dietz V. Recovery of bladder function in patients with acute spinal cord injury: significance of ASIA scores and somatosensory evoked potentials. Spinal Cord 1997; 35: $368-373$.

13 Brandstater ME, Dinsdale SM. Electrophysiological studies in the assessment of spinal cord lesions. Arch Phys Med Rehabil 1976; 57: 70-74.

14 Campbell JW et al. Spontaneous electromyographic potentials in chronic spinal cord injured patients: relation to spasticity and length of nerve. Arch Phys Med Rehabil 1991; 72: 23-27.

15 Laurence TN, Pugel AV, Teasdall RD. Peripheral nerve involvement in spinal cord injury: An electromyographic study. Arch Phys Med Rehabil 1978; 59: 303-313.

16 Krasilovsky G. Nerve conduction studies in patients with cervical spinal cord injury. Arch Phys Med Rehabil 1980; 61: $204-209$.

17 Stark RJ, Kennard C, Swash M. Hand wasting in spondylotic high cord compression: An electromyographic study. Ann Neurol 1981; 9: $58-62$.

18 Takebe K, Narayan MG, Kukulka C, Basmajian JV. Slowing of nerve conduction velocity in hemiplegia: possible factors. Arch Phys Med Rehabil 1975; 56: 285-289.

19 Goldkamp O. Electromyography and nerve conduction studies in 116 patients with hemiplegia. Arch Phys Med Rehabil 1967; 48: $59-63$. 
20 Segura RP, Sahgal V. Hemiplegic atrophy: electrophysiological and morphological studies. Muscle Nerve 1981; 4: 246-248.

21 McComas AJ, Sica REP, Upton ARM, Aquilera N. Functional changes in motoneurons of hemiparetic patients. $J$ Neurol Neurosurg Psychiatry 1973; 36: $183-193$.

22 Nyboer VJ, Johnson HE. Electromyographic findings in lower extremities of patients with traumatic quadriplegia. Arch Phys Med Rehabil 1971; 6: 256-259.

23 O'Hare JM, Abbort GH. Electromyographic evidence of lower motoneuron in cervical spinal cord injury. Spinal Cord injury Conf 1967; 16: 25 - 27.

24 Rosen JS, Lerner IM, Rosenthal AM. Electromyography in spinal cord injury. Arch Phys Med Rehabil 1969; 50: 271 - 273

25 Berman SA, Young RR, Sarkarati M, Shefner JM. Injury zone denervation in traumatic quadriplegia in humans. Muscle Nerve 1996; 19: $701-706$.

26 Blaik Z, McGary J, Daura R. Peripheral neuropathy in spinal cord injured patients. Electromyogr Clin Neurophysiol 1989; 29: $469-472$.

27 Hunter J, Ashby P. Secondary changes in segmental neurons below a spinal cord lesion in man. Arch Phys Med Rehabil 1984; 65: $702-705$.

28 Taylor S, Ashby P, Verrier M. Neurophysiological changes following a traumatic spinal cord lesion in man. J Neurol Neurosurg Psychiatry 1984; 47: $1102-1108$.
29 Taylor RH, Kewalramani LS, Fowler Jr WM. Electromyographic findings in lower extremities of patients with high spinal cord injury. Arch Phys Med Rehabil 1974; 55: 16-23.

30 Benecke R, Berthold A. Conrad B. Denervation activity in the EMG of patients with upper motor neuron lesions: time course, local distribution and pathogenic aspects. J Neurol 1983; 230: $143-151$.

31 Aisen ML, Brown W, Dubin M. Electrophysiological changes in lumbar spinal cord after cervical spinal cord injury. Neurology 1992; 42: $623-626$.

32 Curt A, Keck M, Dietz V. Clinical value of F-wave recordings in traumatic cervical spinal cord injury. Electroenceph Clin Neurophysiol 1997; 105: 189-193.

33 Ditunno JF, Stover SL, Fried MM, Ahn JH. Motor recovery of the upper extremities in traumatic quadriplegia: a multicenter study. Arch Phys Med Rehabil 1992; 73: 431-436.

34 Crozier KS, Lingling Cheng BS, Graziani V, Zorn G, Herbison G, Ditunno JF. Spinal cord injury: prognosis for ambulation based on quadriceps recovery. Paraplegia 1992; 30: $762-767$

35 Curt A, Keck ME, Dietz V. Functional outcome following spinal cord injury: significance of motor-evoked potentials and ASIA scores. Arch Phys Med Rehabil 1997; 79: 81-86. 